The Astrophysical Journal, 228:405-418, 1979 March 1

(C) 1979. The American Astronomical Society. All rights reserved. Printed in U.S.A.

\title{
OBSERVATIONS AND INTERPRETATIONS OF RADIAL GRADIENTS OF ABSORPTION FEATURES IN GALAXIES
}

\author{
JUDITH G. COHEN \\ Kitt Peak National Observatory* \\ Received 1978 May 1; accepted 1978 September 7
}

\begin{abstract}
Observations are presented of equivalent widths for strong spectral features with $\lambda>5000 \AA$ in the nuclei of five galaxies (M31, M32, M81, NGC 4472, and NGC 3115) as well as the radial gradients of line strength within these galaxies. A theoretical model for predicting the strengths of features in the integrated light of galaxies is also presented. It is concluded, on the basis of this limited sample of galaxies, that the changes in spectral features between galactic nuclei and within galaxies arise from a single causal factor, which is the overall metallicity of the galaxy. The range in nuclear abundance spanned by the five galaxies is a factor of 4 . There is no evidence for nonsolar ratios among the elements with lines that have been studied, namely, $\mathrm{Ca}, \mathrm{Na}$, $\mathrm{Mg}$, and $\mathrm{Fe}$; specifically, the approximate constancy of the $\mathrm{Fe}$ blends within and between galaxies has a natural explanation. Furthermore, the nuclear metallicity ordering is the same as the ordering of $M_{v}$.
\end{abstract}

Subject headings: abundances - galaxies: nuclei — galaxies: stellar content

\section{INTRODUCTION}

Our present conceptions of the formation and chemical history of galaxies imply the buildup of the heavy elements as the galaxy collapses. Therefore a metallicity gradient in galaxies, except perhaps in irregulars and other very low-mass systems, is often assumed to exist as a function of radius for some or most elements, with the highest metallicity being found in the nucleus itself. The existence of this metallicity gradient has been inferred from observed color gradients in galaxies (e.g., de Vaucouleurs 1961; Tifft 1969; Strom et al. 1976), and a calibration of expected integrated color as a function of metallicity has been given by Aaronson et al. (1978, hereafter ACMM). Observations of line-strength gradients are much more difficult and hence much fewer. With the use of narrow-band photometry, radial gradients have been detected, principally in $\mathrm{CN}$ and in the $\mathrm{Na} \mathrm{I}$ doublet 5889-5895 § (McClure 1969; Spinrad et al. 1971; Spinrad, Smith, and Taylor 1972). Gradients in line strength based on image-tube spectra treated as photometric indices have been given by Welch and Forrester (1972), while Joly and Andrillat (1973) and Faber (1977) present equivalent width measurements based on photographic and photoelectric spectra, respectively. The previous results have, in general, been restricted to wavelengths bluer than $6000 \AA$.

In order to separate metallicity enhancements from possible changes in the composite population, we present in $\S$ II observational data taken with a multielement linear array detector for the lines $\mathrm{Na} I$ (5889-5895 $\AA$; denoted Na D), Na I (8183-8195 $\AA$;

* Operated by the Association of Universities for Research in Astronomy, Inc., under contract with the National Science Foundation. denoted $\mathrm{Na}$ IR), $\mathrm{Ca}$ II $(8498,8542$, and $8662 \AA$; denoted Ca T), Mg I (5169-5183 $\AA$; denoted Mg b), and other weaker blends, principally of $\mathrm{Fe}$, for M31, M32, and NGC 3115, and, for a limited set of observational data, M81 and NGC 4472. In § III a theoretical model of the spectral features in the integrated light is developed, based on the models of ACMM. In § IV we consider the interpretation, in a manner similar to that of Cohen (1978), of the results for the nuclei alone, while in $\S \mathrm{V}$ we try to determine from the observed radial line gradients which (if not all) elements are varying in abundance.

\section{OBSERVATIONS}

The data were acquired with a $100 \times 100$ pixel CID array in a Dewar cooled to liquid nitrogen on the gold spectrograph at the Kitt Peak National Observatory $2.1 \mathrm{~m}$ telescope, with the Nye lens as a camera, during four nights in 1977 November. The original dispersion was $83 \AA \mathrm{mm}^{-1}$, which translates to $5.2 \AA$

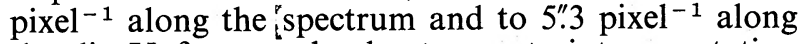
the slit. Unfortunately, due to constraints on rotation of the spectrograph, the slit was always oriented in the east-west direction and could not be aligned with the major or minor axes of the galaxies. The full width at half-maximum of neon or argon comparison lines was 1.2 pixels. These spectra are identical in character to those of class C of Cohen (1978), where a description of the CID system can be found, although a significant improvement in the electronic stability of the device has occurred in the interim.

The reduction of these spectra proceeded in two steps. We note that, because of imprecise centering of the array with respect to the projected spectrograph decker, an area 30 columns wide was beyond the edge 


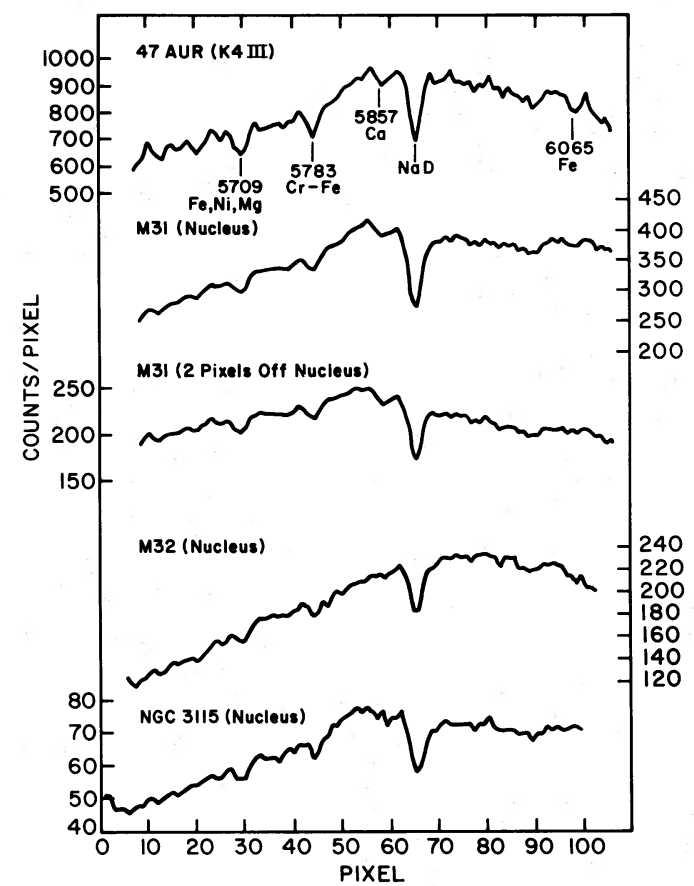

FIG. 1.-Representative CID data are shown for a $\mathrm{K}$ giant and three galaxies. The counts per pixel are shown for each object, and the vertical scale is approximately the same in residual intensity for each spectrum. The scan of 47 Aur is the average of two columns of a single frame, as are the M31 and NGC 3115 scans. The M32 spectrum is from a single column of one frame.

of the large decker used here and hence not exposed to any light. The spectrum for a star was 2.5 columns wide at FWHM, and its center shifted by less than 1 column over the entire array from row 1 to row 100. First, the no-light frame was subtracted from the picture, then the whole array was shifted by a constant so that no light corresponded to zero counts (i.e., the mean electronic drift problems averaged over the entire array were removed, although the dark count, as such, is so small that it cannot be detected). The frames were then divided by the flat-field calibration taken with a quartz iodide lamp. Finally, the remaining odd-even effect was removed by using an algorithm from the approximately 30 columns $\times 100$ rows which had not been exposed to light (usually fewer than 10 counts). This part of the reduction, which we may consider the conversion to a linear intensity scale, was performed on the KPNO Interactive Picture Processing System. Representative spectra of a K giant and three galaxies obtained from single frames are shown in Figure 1. The high signal-to-noise ratio of the data is apparent and is a result of the A/D conversion being set at one count per 500 electrons.

The next phase was the measurement of the strength of the spectral features. By using a minicomputer and a graphics terminal, we measured the equivalent width of each feature on each column of the picture that had more than 30 counts. Naturally the weaker features could not be measured at such low count levels. For M31 only, adjacent columns were averaged, and then the features were measured. Thus the equivalent widths here are numerical integrations of the area within the feature where the continuum is represented by a straight line between two points, one on each side of the feature. In practice, because we have avoided the extremely crowded blue region of the spectrum, the choice of the continuum was not a problem until count rates fell below 50, at which time some residual electronic problems in the CID array became apparent.

In Table 1 we list the pictures available for each spectral region, where the exposure time and the count rate of a pixel centered on the nucleus at a continuum wavelength for each picture are also indicated. Tables $2 \mathrm{~A}-2 \mathrm{C}$ list the measured equivalent widths (in $\AA$ ) as a function of pixels from the nucleus, where separate measurements of $W_{\lambda}$ from each available picture have been averaged. (The number of frames for each line is indicated in the third row.) Values in parentheses are given where the feature was not measured on one of the two or two of the three available pictures at that offset from the nucleus. A continuum light profile at $5900 \AA$, as a function of pixels from the nucleus, is included in Table 3 for each galaxy.

TABLE 1

JOURNAL OF OBSERVATIONS

\begin{tabular}{|c|c|c|c|c|c|c|c|c|c|c|}
\hline \multirow{2}{*}{$\begin{array}{c}\text { SPECTRAL } \\
\text { REGION } \\
(\AA)\end{array}$} & \multicolumn{2}{|c|}{ M31 } & \multicolumn{2}{|c|}{ M32 } & \multicolumn{2}{|c|}{ NGC 3115} & \multicolumn{2}{|c|}{ M81 } & \multicolumn{2}{|c|}{ NGC 4472} \\
\hline & $\begin{array}{l}\text { Time } \\
\text { (min) }\end{array}$ & $\begin{array}{c}\text { Max. } \\
\text { Count* }\end{array}$ & $\begin{array}{l}\text { Time } \\
\text { (min) }\end{array}$ & $\begin{array}{l}\text { Max. } \\
\text { Count }\end{array}$ & $\begin{array}{l}\text { Time } \\
\text { (min) }\end{array}$ & $\begin{array}{l}\text { Max. } \\
\text { Count }\end{array}$ & $\begin{array}{l}\text { Time } \\
\text { (min) }\end{array}$ & $\begin{array}{l}\text { Max. } \\
\text { Count }\end{array}$ & $\begin{array}{l}\text { Time } \\
\text { (min) }\end{array}$ & $\begin{array}{l}\text { Max. } \\
\text { Count }\end{array}$ \\
\hline $\begin{array}{l}5000-5500 \ldots \\
5600-6100 \ldots\end{array}$ & $\begin{array}{l}50 \\
25 \\
35\end{array}$ & $\begin{array}{l}170 \\
225 \\
310\end{array}$ & $\begin{array}{l}25 \\
15 \\
15\end{array}$ & $\begin{array}{l}115 \\
185 \\
145\end{array}$ & $\begin{array}{l}50 \\
40 \\
35\end{array}$ & $\begin{array}{l}40 \\
90 \\
75\end{array}$ & $\begin{array}{l}25 \\
\ldots\end{array}$ & $\begin{array}{l}155 \\
\ldots\end{array}$ & $\begin{array}{l}35 \\
\cdots\end{array}$ & $\begin{array}{l}55 \\
\cdots\end{array}$ \\
\hline $8050-8550$ & 45 & 420 & & & $\cdots$ & $\cdots$ & $\cdots$ & $\cdots$ & $\cdots$ & $\cdots$ \\
\hline $8050-8550 \ldots$ & 35 & 265 & $\begin{array}{l}45 \\
30\end{array}$ & $\begin{array}{l}290 \\
235\end{array}$ & $\cdots$ & $\cdots$ & $\cdots$ & $\cdots$ & $\cdots$ & $\cdots$ \\
\hline $8300-8800 \ldots$ & $\begin{array}{l}30 \\
25\end{array}$ & $\begin{array}{l}125 \\
170\end{array}$ & $\begin{array}{l}30 \\
25\end{array}$ & $\begin{array}{l}190 \\
190\end{array}$ & $\begin{array}{l}50 \\
35\end{array}$ & $\begin{array}{l}50 \\
65\end{array}$ & $\begin{array}{r}30 \\
\ldots\end{array}$ & $\begin{array}{r}95 \\
\ldots\end{array}$ & $\begin{array}{r}20 \\
\ldots\end{array}$ & $\begin{array}{c}25 \\
\ldots\end{array}$ \\
\hline
\end{tabular}

* A/D conversion was always 1 count per 500 electrons. 


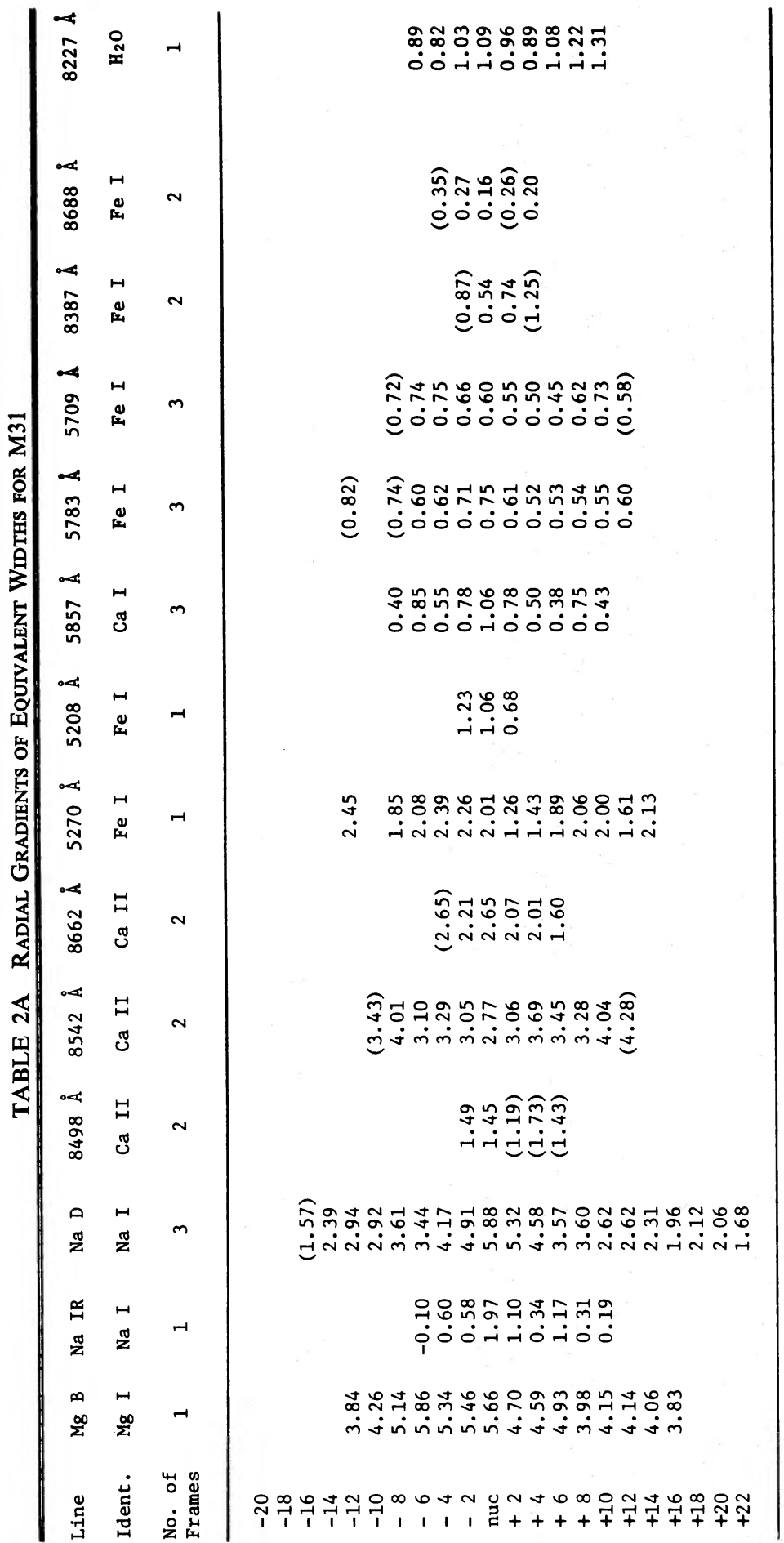

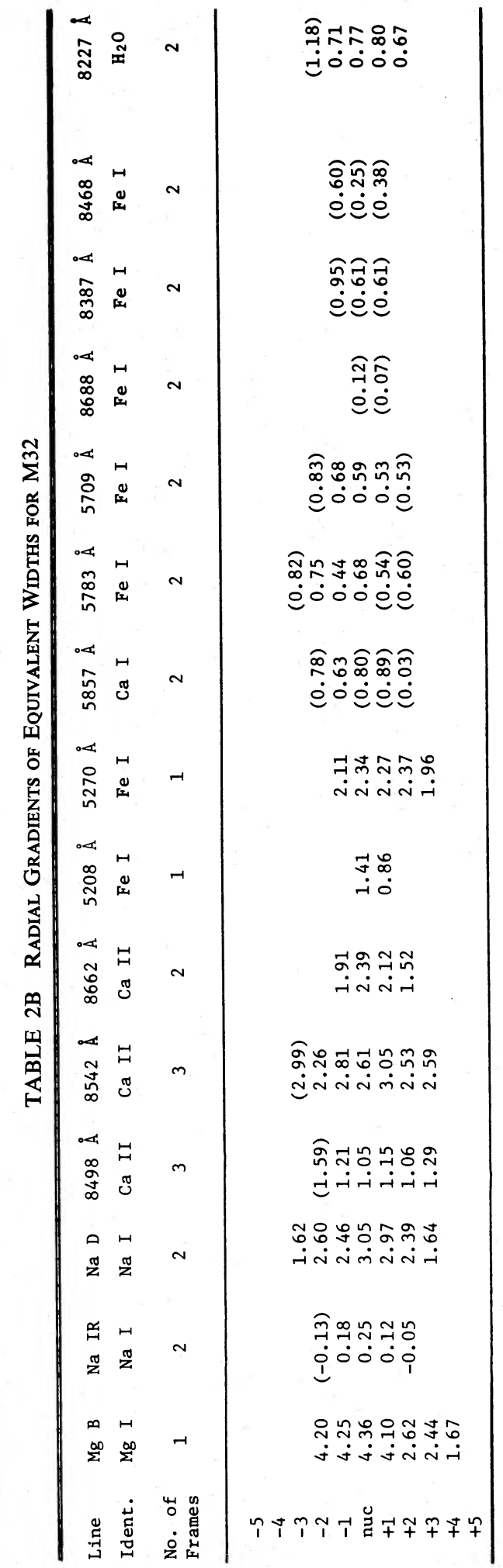


TABLE 2C

Radial Gradients of Equivalent WidTHS fOR NGC 3115, M81, AND NGC 4472

\begin{tabular}{|c|c|c|c|c|c|c|c|c|}
\hline & & & & NGC 3115 & & & & \\
\hline Line & Mg B & Na $\mathrm{D}$ & $8498 \AA$ & $8542 \AA$ & $8662 \AA$ & $5270 \AA$ & $5709 \AA$ & $5783 \AA$ \\
\hline Ident. & Mg I & Na I & $\mathrm{Ca}$ II & $\mathrm{Ca}$ II & $\mathrm{Ca}$ II & Fe I & $\mathrm{Fe} \mathrm{I}$ & $\mathrm{Fe} I$ \\
\hline $\begin{array}{l}\text { No. of } \\
\text { Frames }\end{array}$ & 1 & 2 & 2 & 2 & 2 & 1 & 2 & 2 \\
\hline $\begin{array}{r}-4 \\
-3 \\
-2 \\
-1 \\
\text { nuc } \\
\text { nuc } \\
+1 \\
+2 \\
+3 \\
+4 \\
+5 \\
+6\end{array}$ & $\begin{array}{l}3.68 \\
5.44 \\
4.44 \\
4.63 \\
4.88 \\
5.49 \\
4.80 \\
4.66 \\
3.93 \\
3.32 \\
2.34\end{array}$ & $\begin{array}{c}(3.75) \\
3.69 \\
3.70 \\
4.08 \\
4.57 \\
4.22 \\
3.73 \\
(4.01) \\
(3.25)\end{array}$ & $\begin{array}{c}1.68 \\
1.55 \\
1.80 \\
1.65 \\
(1.65) \\
(2.21)\end{array}$ & $\begin{array}{c}3.30 \\
2.99 \\
3.18 \\
3.40 \\
(2.74) \\
(3.92)\end{array}$ & $\begin{array}{c}2.47 \\
2.53 \\
2.24 \\
(1.97)\end{array}$ & $\begin{array}{l}2.21 \\
1.90 \\
1.02 \\
1.38 \\
1.40 \\
1.63 \\
1.29 \\
1.75 \\
1.83\end{array}$ & $\begin{array}{l}0.57 \\
0.75 \\
(1.08)\end{array}$ & $\begin{array}{c}(0.31) \\
0.47\end{array}$ \\
\hline & & & & $\underline{\mathrm{M} 81}$ & & & & \\
\hline Line & $\mathrm{Na} D$ & $8498 \AA$ & $8542 \AA$ & $8662 \AA$ & $8468 \AA$ & $5857 \AA$ & $5783 \AA$ & $5709 \AA$ \\
\hline Ident. & $\mathrm{Na} \mathrm{I}$ & $\mathrm{Ca}$ II & $\mathrm{Ca}$ II & $\mathrm{Ca}$ II & $\mathrm{Fe} \mathrm{I}$ & $\mathrm{Ca} \mathrm{I}$ & $\mathrm{Fe} \mathrm{I}$ & $\mathrm{Fe} \mathrm{I}$ \\
\hline $\begin{array}{l}\text { No. of } \\
\text { Frames }\end{array}$ & 1 & 1 & 1 & 1 & 1 & 1 & 1 & 1 \\
\hline $\begin{array}{r}-3 \\
-2 \\
-1 \\
\text { nuc } \\
\text { nuc } \\
+1 \\
+2 \\
+3\end{array}$ & $\begin{array}{l}3.87 \\
4.78 \\
4.87 \\
6.47 \\
7.27 \\
6.39 \\
4.91 \\
4.08\end{array}$ & 1.18 & $\begin{array}{l}4.43 \\
3.67 \\
3.62 \\
2.35 \\
2.82 \\
3.02\end{array}$ & $\begin{array}{l}2.18 \\
1.79 \\
2.23 \\
2.18\end{array}$ & 1.22 & $\begin{array}{l}1.23 \\
1.22\end{array}$ & $\begin{array}{l}0.82 \\
0.85 \\
0.74 \\
0.50 \\
0.67 \\
0.52\end{array}$ & $\begin{array}{l}0.90 \\
0.59 \\
0.42 \\
0.51 \\
0.65\end{array}$ \\
\hline
\end{tabular}

\begin{tabular}{lccccc}
\multicolumn{8}{c}{ NGC 4472} & \\
Line & Na D & $8498 \AA$ & $5842 \AA$ & $8662 \AA$ & $5783 \AA$ \\
Ident. & Na I & Ca II & Ca II & Ca II & Fe I \\
No. of & 1 & 1 & 1 & 1 & 1 \\
Frames & 1 & & & & \\
\hline-4 & 5.57 & & & \\
-3 & 6.23 & & & \\
-2 & 5.49 & & & \\
-1 & 5.58 & & & & \\
nuC & 5.70 & 2.14 & 2.97 & \\
+1 & 5.59 & & & & \\
+2 & 5.27 & & & & \\
+3 & 6.18 & & & & \\
+4 & 4.59 & & & & \\
\hline
\end{tabular}

The infrared $\mathrm{Na}$ doublet was corrected as accurately as possible for the presence of atmospheric $\mathrm{H}_{2} \mathrm{O}$ absorption by using $W_{\lambda}$ for terrestrial features measured from CID array spectra of B stars and of a few early $\mathrm{K}$ giants. The accuracy of the $\mathrm{Na}$ IR measurements is less than that of other features because of the substantial contribution of terrestrial absorption to the observed absorption in the galaxy spectra.

An indication of the accuracy of the entire process can be obtained by considering $W_{\lambda}$ of the water-vapor blend at $8227 \AA$, which is also included in Table 2 . This feature, with $W_{\lambda}=0.8-1.0 \AA$, depending on the night considered, displayed a $25 \%$ variation in $W_{\lambda}$ over the center 20 pixels of M31 from only one picture. $W_{\lambda}$ for the stronger features is thus expected to be accurate to $\pm 15 \%$ from two pictures, except for $\mathrm{Na}$ IR.

III. A MODEL FOR SPECTRAL FEATURES IN THE INTEGRATED LIGHT

a) The Population Model and $W_{\lambda}$ Calibrations

We develop here a scheme for predicting the strength of absorption features in the integrated light 
TABLE 3

Continuum Light Profiles at $5900 \AA$

\begin{tabular}{|c|c|c|c|c|c|c|}
\hline Pixel & M31 & Pixel & M32 & NGC 3115 & NGC 4472 & M81 \\
\hline 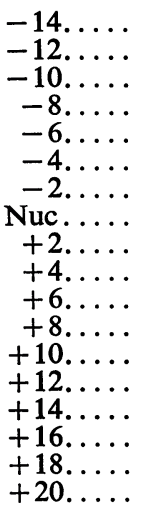 & $\begin{array}{l}0.11 \\
0.14 \\
0.17 \\
0.21 \\
0.27 \\
0.37 \\
0.58 \\
1.00 \\
0.84 \\
0.54 \\
0.43 \\
0.36 \\
0.31 \\
0.29 \\
0.24 \\
0.21 \\
0.19 \\
0.16\end{array}$ & $\begin{array}{l}-5 \ldots \ldots \\
-4 \ldots \ldots \\
-3 \ldots \ldots \\
-2 \ldots \ldots \\
-1 \ldots \ldots \\
\text { Nuc. } \\
+1 \ldots \\
+2 \ldots \\
+3 \ldots \\
+4 \ldots \\
+5 \ldots\end{array}$ & $\begin{array}{l}\ldots \\
\ldots .12 \\
0.27 \\
0.58 \\
1.00 \\
0.74 \\
0.46 \\
0.18 \\
\ldots \\
\ldots\end{array}$ & $\begin{array}{l}0.26 \\
0.53 \\
0.71 \\
0.89 \\
0.95 \\
1.00 \\
0.87 \\
0.72 \\
0.46 \\
0.29 \\
\ldots\end{array}$ & $\begin{array}{l}0.32 \\
0.47 \\
0.72 \\
0.88 \\
1.00 \\
0.89 \\
0.76 \\
0.52 \\
0.37 \\
\ldots\end{array}$ & $\begin{array}{l}0.14 \\
0.27 \\
0.55 \\
0.94 \\
1.00 \\
0.76 \\
0.44 \\
0.23 \\
\ldots \\
\ldots\end{array}$ \\
\hline
\end{tabular}

of a galaxy. This requires two basic components: a population model, and a relationship between stellar parameters and $W_{\lambda}$ for the spectral features. The population model is that of ACMM, which is based on the evolutionary tracks of Ciardullo and Demarque (1977). A single burst of star formation $13 \times 10^{9}$ years ago is assumed. The population model is a function of metallicity $Z$ (where $Z_{\odot}=1.0$ ) and also depends on the slope of the initial mass function $s$, where $s=1+x$ in the notation of Tinsley (1972). A helium content $Y=0.30$ is assumed. Further details of the population model and predicted integrated broad-band colors from $U$ to $K$ can be found in ACMM. Since in this model there are no young stars, we cannot expect to successfully apply our scheme to spectral features shortward of $5000 \AA$ where one might expect the Population I contribution to the integrated light to be both nonnegligible and a function of distance from the galaxy nucleus.

The problem of predicting $W_{\lambda}$ from the basic stellar parameters is divided into two parts by first considering the variation in $W_{\lambda}$ at solar metallicity as $T_{\text {eff }}$ and $g$ vary (i.e., in bright, normal field giants and dwarfs) and then evaluating separately the effect of varying $Z$. The spectral features we are considering at this time are $\mathrm{Na} \mathrm{D}, \mathrm{Na} \mathrm{IR}, \mathrm{Mg} b, \mathrm{FeH}$ (the band at $9910 \AA$ ), and $\Sigma W_{\lambda}$ for the three lines of the nearinfrared $\mathrm{Ca}$ II triplet (denoted $\mathrm{Ca} \mathrm{T}$ ). The strengths of these lines are expressed as functions of $T_{\text {eff }}$, with a separate parametrization for giants and for dwarfs. The source of the observational data for the functions used to predict the strength of each feature is indicated below.

$\mathrm{Na}$ D.-The calibration of $\mathrm{Na} \mathrm{D}$ is taken from a compilation by Tinsley (1967) with spectral types converted to $T_{\text {eff }}$ via Johnson (1966). A limited number of bright $\mathrm{K}$ giants were observed with the CID, and the $W_{\lambda}$ for their $\mathrm{D}$ lines agrees with the calibration to within $\pm 0.4 \AA$ for each star.

$\mathrm{Na} I \mathrm{R}$ and $\mathrm{FeH}$.- The calibration of $\mathrm{Na}$ IR and
$\mathrm{FeH}$ is taken from Cohen (1978). ${ }^{1}$ The strength of $\mathrm{Na}$ IR and $\mathrm{FeH}$ in stars with $V-K<4.0$ is negligible and therefore these data alone are sufficient. Note that these observations were secured largely with a CID, and hence there is no question of matching resolutions between the calibration spectra and the CID spectra. For $\mathrm{FeH}$ we calibrate the percent of absorption at $9920 \AA$ at a resolution comparable to that of the CID by using the observations of Cohen (1978).

$\mathrm{Mg} b$.- The calibration of $\mathrm{Mg} b$ for stars hotter than $T_{\text {eff }}=5600 \mathrm{~K}$ was taken from the compilation of Tinsley (1967). For cooler stars, Figure 2 of Mould (1978) was used. Since this refers to a narrow-band photometric index, the predicted $W_{\lambda}$ for $\mathrm{Mg} b$ were scaled by a constant (which was 1.6) to fit the solar $W_{\lambda}$ (Moore, Minnaert, and Houtgast 1966). This scaling constant also makes the predicted $\mathrm{Mg} b$ agree well with the CID measurements of spectra of a few bright $\mathrm{K}$ giants.

$\mathrm{Ca} \mathrm{T}$.- The calibration of $\mathrm{Ca} \mathrm{T}$ as a function of $T_{\text {eff }}$ for giants and dwarfs is taken from Cohen (1978), which is mostly at a resolution comparable to the CID galaxy observations. Unfortunately, this leaves a large gap for stars earlier than $\mathrm{K} 0$ where the $\mathrm{Ca} \mathrm{T}$ is still quite strong. This gap was filled by an educated guess combined with the solar data from Moore et al. Fortunately, the contribution to the integrated light at $8500 \AA$ from dwarfs of spectral types earlier than the Sun is not very significant, and the available observations indicate that giants reach their maximum $\mathrm{Ca} \mathrm{T}$ strength at a $T_{\text {eff }}$ within the range covered by the data of Cohen (1978).

1 The author now realizes that the class $\mathrm{C}$ data only for $W_{\lambda}$ of the infrared $\mathrm{Na}$ I doublet in Cohen (1978) contain an inadequate correction for terrestrial $\mathrm{H}_{2} \mathrm{O}$ absorption. The following modifications were made here to the data in Table 1 of Cohen (1978): +04 $4048\left(W_{\lambda}-1.0 \AA\right) ;+45^{\circ} 2505$ $\left(W_{\lambda}-1.0 \AA\right)$; YZ CMi $\left(W_{\lambda}-0.3 \AA\right)$; Wolf $359\left(W_{\lambda}-\right.$ $0.5 \AA \AA)$. 


\section{b) Metallicity Effects for Strong Lines}

We can now use the predicted contribution to the integrated light at a continuum point near each spectral feature from our population model combined with the $W_{\lambda}$ calibrations described above to predict the $W_{\lambda}$ in the integrated light of various spectral features if the stars were of solar metallicity, where $W_{\lambda}$ in the integrated light is given by $\Sigma W_{\lambda}\left(T_{\text {eff }}, M_{\text {bol }}\right) \times$ $\mathrm{CF}\left(\lambda, T_{\text {eff }}, M_{\mathrm{bol}}\right)$, and CF (the contribution function) is determined from the population model and is the percentage contribution to the integrated light in the continuum at a given $\lambda$ by stars in each $T_{\text {eff }}$ and $M_{\text {bol }}$ increment. Note that we can do this for varying $Z$ insofar as metallicity affects the mean-sequence and giant-branch $T_{\text {eff }}$ and luminosity, but we cannot yet take into account the effect of varying $Z$ on the $W_{\lambda}$ of an individual star. The results of such calculations for $Z=0.5$ and 2.0 are given in Table 4 for $s=0$, 2.35 , and 4.0. To aid in computing the necessary contribution functions, the galaxy models of ACMM were extended to include the Johnson (1966) $I$ color $\left(\lambda_{\text {eff }} \approx 9000 \AA\right)$ by using his $V-I$ versus $V-K$ calibrations. The adopted contribution functions were as follows: that of $V$ for $\mathrm{Na} \mathrm{D}$ and $\mathrm{Mg} b$, a weighted mean between $V$ and $I$ for Na IR, a mean between $V$ and $I$ weighted heavily toward $I$ for $\mathrm{Ca} \mathrm{T}$, and a weighted mean between $I$ and $J$ for $\mathrm{FeH}$. The good fits (except in $U-V$ ) obtained from the population model of ACMM to integrated colors of globular clusters and nuclei of early-type galaxies give us great confidence in the accuracy of our contribution functions, since we do not require them at any blue or ultraviolet wavelengths.

We must now consider the effect of varying $Z$ on the $W_{\lambda}$ for an individual giant or dwarf of fixed $T_{\text {eff }}$. We shall follow the approach of Cohen (1978) and define, for each line $i$ under consideration, the metal- licity multiplication factor ${ }^{2} \mathrm{MMF}(i)$,

$$
\frac{W_{\lambda}^{i}(Z)}{W_{\lambda}^{i}(Z=1)}=(Z)^{\mathrm{MMF}(i)}=(Z)^{\alpha}\left(\frac{g_{Z}}{g_{Z=1}}\right)^{\beta},
$$

where $g_{z}$ is the surface gravity, which varies for a fixed $T_{\text {eff }}$ due to the shift of the giant branch in luminosity and mass as $Z$ is changed. (We assume either no mass loss or a mass loss proportional to the original mass here.)

All the lines of interest are very strong over the range of $T_{\text {eff }}$ that contributes significantly to the integrated light, and furthermore we are considering only a small range in $Z$, from 0.5 to 2.0 . Hence we will assume that all the lines under consideration are on the damping part of the curve of growth all the time. This assumption is worse, but still acceptable, for $\mathrm{Na} \mathrm{IR,} \mathrm{as} \mathrm{it} \mathrm{is} \mathrm{the} \mathrm{weakest} \mathrm{feature} \mathrm{considered.} \mathrm{We}$ may then obtain purely theoretical values for the MMFs from Cayrel and Jugaku (1963) and from Deutsch (1966), by using the model-atmosphere calculations for $\mathrm{K}$ giants of Cohen, Frogel, and Persson (1978) as a guide to the ionization of the relevant ions. To evaluate $g_{z}$, we note that the predicted change along the giant branch in $M_{\text {bol }}$ for a fixed $T_{\text {eff }}$ from $Z=0.5$ to $Z=2.0$ from Ciardullo and Demarque (1977) is $\sim 0.9 \mathrm{mag}$, and the mass changes by a factor of 1.15 , so that the resulting change in surface gravity is approximately 0.40 dex or a factor of 2.5 . Although the values of $\alpha$ and $\beta$ given by Cayrel and Jugaku (1963) and by Deutsch (1966) differ slightly, and one cannot represent the changes in giants and in dwarfs by exactly the same values, since in the first case the

\footnotetext{
${ }^{2}$ Note that the MMF tabulated in Cohen (1978) included a correction for the shift of the giant branch in $T_{\text {eff }}$ as $Z$ varied, whereas this factor is separately treated in the more
} detailed and exact analysis presented here.

TABLE 4

Predicted Strengths* of Spectral Features in INTEgRated Light USing Solar Metallicity $W_{\lambda}$ Calibrations

\begin{tabular}{|c|c|c|c|c|c|c|}
\hline & $\begin{array}{l}\text { Feature } \\
\text { Strength }\end{array}$ & $\begin{array}{c}\mathrm{FeH} \\
(\% \text { abs.) }\end{array}$ & $\underset{\left(W_{\lambda}[\AA \AA]\right)}{\mathrm{Na} \mathrm{D}}$ & $\underset{\left.\left(W_{\lambda}[\AA]\right]\right)}{\mathrm{Na} \mathrm{IR}}$ & $\begin{array}{c}\mathbf{M g} b \\
\left.\left(W_{\lambda}[\AA]\right]\right)\end{array}$ & $\underset{\left(W_{\lambda}[\AA ̊ \AA]\right.}{\mathrm{Ca}]}$ \\
\hline \multicolumn{7}{|c|}{$Z=2$} \\
\hline$s$ & $\begin{array}{l}0.0 \\
2.35 \\
4.0\end{array}$ & $\begin{array}{l}1.5 \\
2.4 \\
9.3\end{array}$ & $\begin{array}{l}3.60 \\
4.12 \\
6.05\end{array}$ & $\begin{array}{l}0.13 \\
0.28 \\
1.76\end{array}$ & $\begin{array}{l}5.12 \\
5.26 \\
5.58\end{array}$ & $\begin{array}{l}6.80 \\
6.45 \\
4.79\end{array}$ \\
\hline \multicolumn{7}{|c|}{$Z=0.5$} \\
\hline$s$ & $\begin{array}{l}0.0 \\
2.35 \\
4.0\end{array}$ & $\begin{array}{l}0.4 \\
1.2 \\
7.4\end{array}$ & $\begin{array}{l}2.96 \\
3.89 \\
7.37\end{array}$ & $\begin{array}{l}0.03 \\
0.16 \\
1.27\end{array}$ & $\begin{array}{l}4.37 \\
4.64 \\
5.41\end{array}$ & $\begin{array}{l}6.99 \\
6.47 \\
4.70\end{array}$ \\
\hline
\end{tabular}

* Includes shift of giant branch but not effect of varying $Z$ on spectra of stars of constant $T_{\text {eff }}$; see test for detailed explanation. 
TABLE 5

MMF VALUES

\begin{tabular}{|c|c|c|c|c|c|}
\hline \multirow[b]{2}{*}{ LINE } & \multirow[b]{2}{*}{$\begin{array}{l}\text { SIMPLE THEORY* } \\
\text { MMF }\end{array}$} & \multicolumn{2}{|c|}{ OBSERVATIONS } & \multirow{2}{*}{$\begin{array}{c}\text { MODEL- } \\
\text { ATMOSPHERE } \\
\text { PREDICTIONS }\end{array}$} & \multirow[b]{2}{*}{ ADOPTED } \\
\hline & & MMF & $\begin{array}{l}\text { No. of } \\
\text { Stars }\end{array}$ & & \\
\hline$\ldots \ldots \ldots \ldots \ldots \ldots$ & 0.5 & $0.45 \pm 0.2$ & 7 & $\ldots$ & 0.45 \\
\hline $\mathrm{Na} \mathrm{IR} \ldots \ldots \ldots \ldots \ldots \ldots \ldots$ & 0.5 & & & $\ldots$ & 0.45 \\
\hline Ca $\mathbf{T} \ldots \ldots \ldots \ldots \ldots \ldots \ldots$ & 0.0 & $0.2 \pm 0.1$ & $\ddot{2}$ & $\ldots$ & 0.1 \\
\hline $\mathrm{FeH}, \ldots \ldots \ldots \ldots \ldots \ldots \ldots$ & 0.5 & & & & 0.45 \\
\hline $\operatorname{Mg} b \ldots \ldots \ldots \ldots \ldots \ldots \ldots$ & $0.0-0.5$ & 0.25 & $i$ & $0.22 \pm 0.05 \dagger$ & 0.22 \\
\hline Damped Fe $1 . \ldots \ldots \ldots \ldots$ & $0.0-0.5$ but less than $\mathrm{Mg} b$ & $\ldots$ & $\ldots$ & $\ldots$ & 0.10 \\
\hline Weak Fe $1, \ldots \ldots \ldots \ldots \ldots$ & $0.5-1.0$ & $\ldots$ & $\ldots$ & $\ldots$ & $\ldots$ \\
\hline
\end{tabular}

* Cayrel and Jugaku 1963 and Deutsch 1966.

$\dagger$ Mould 1978.

damping is mostly radiative, while in the second it is collisional, the values listed in the first column of Table 5 represent reasonable approximations for the MMF values as deduced from purely theoretical parametrizations of the relevant opacities. As Fe is more neutral than $\mathrm{Mg}$ throughout the $\mathrm{K}$ giant atmosphere, we obtain the theoretical values given in Table 5 for later use for an Fe I line on the damping part of the curve of growth and a weak Fe $\mathrm{I}$ line on the linear part of the curve of growth.

A second approach is to use observations of metal-poor giants and subdwarfs, for which a detailed abundance analysis is available, and derive empirical values of $\mathrm{MMF}(i)$ from $W_{\lambda}$ in these stars compared to $W_{\lambda}$ predicted by our $Z=1$ calibrations at the same $T_{\text {eff }}$. Obviously, one must avoid stars so metaldeficient that the feature becomes too weak and it is no longer on the damping part of the curve of growth, but in practice this is not a serious problem except for $\mathrm{Fe} I$ features. Unfortunately, the amount of suitable observational data is severely limited for the near-infrared spectral features; one obtains the results listed in column (2) of Table 5. The single entry in the final column lists the MMF derived from Table 1 of Mould (1978) based on synthetic spectra for a grid of model atmospheres of varying $Z$. An observational and computational effort to fill in the gaps in Table 5 would be very useful. At present the limited available data are in good agreement with the predictions of simple theory (column [1]). We therefore adopt the numbers in the final column of Table 5 for later use. We apply the adopted MMF values to the tabulations of Table 4 to obtain the final predicted strengths of spectral features in integrated light shown in Table 6.

\section{c) Weaker Features (Including Fe I Blends)}

At this point we should note that the extension to features of moderate strength $(0.3-1.0 \AA)$ of the procedure described above is not straightforward. This is because the assumption that the feature is on the damping part of the curve of growth all the time (or at least all the time that the contribution function is large) is no longer valid, unless the feature has zero strength in giants and is very strong in late-type dwarfs (i.e., Na IR). For moderately strong features (such as those of $\mathrm{Fe} I$ ) without the strong gravity dependence described above, the MMF are not constant and must be expressed as functions of $W_{\lambda}$. Because of the importance of weaker blends, specifically those of $\mathrm{Fe} \mathrm{I}$, in determining which elements actually are varying, we must explore this problem.

The most straightforward way to consider the metallicity variation of weak $(0.2-1.0 \AA)$ Fe I blends,

TABLE 6

Predicted Strengths of Spectral Features in Integrated Light

\begin{tabular}{|c|c|c|c|c|c|c|}
\hline & $\begin{array}{l}\text { Feature } \\
\text { Strength }\end{array}$ & $\begin{array}{c}\mathrm{FeH} \\
(\% \text { abs. })\end{array}$ & $\underset{\left.\left(W_{\lambda}[\AA]\right]\right)}{\mathrm{Na} D}$ & $\underset{\left(W_{\lambda}[\AA]\right)}{\mathrm{Na} \operatorname{IR}}$ & $\begin{array}{c}\operatorname{Mg} b \\
\left(W_{\lambda}[\AA]\right)\end{array}$ & $\underset{\left(W_{\lambda}[\AA ̊]\right.}{\mathrm{Ca}}$ \\
\hline \multicolumn{7}{|c|}{$Z=2$} \\
\hline$s$ & $\begin{array}{l}0.0 \\
2.35 \\
4.0\end{array}$ & $\begin{array}{r}2.0 \\
3.3 \\
10.1\end{array}$ & $\begin{array}{l}4.92 \\
5.63 \\
8.26\end{array}$ & $\begin{array}{l}0.18 \\
0.39 \\
2.40\end{array}$ & $\begin{array}{l}5.96 \\
6.13 \\
6.50\end{array}$ & $\begin{array}{l}7.29 \\
6.91 \\
5.13\end{array}$ \\
\hline \multicolumn{7}{|c|}{$Z=0.5$} \\
\hline$s$ & $\begin{array}{l}0.0 \\
2.35 \\
4.0\end{array}$ & $\begin{array}{l}0.3 \\
0.9 \\
5.4\end{array}$ & $\begin{array}{l}2.17 \\
2.85 \\
5.40\end{array}$ & $\begin{array}{l}0.02 \\
0.09 \\
0.93\end{array}$ & $\begin{array}{l}3.75 \\
3.98 \\
4.65\end{array}$ & $\begin{array}{l}6.52 \\
6.04 \\
4.39\end{array}$ \\
\hline
\end{tabular}


TABLE 7

$W_{\lambda}$ FOR WEAK LINES

\begin{tabular}{|c|c|c|c|c|c|}
\hline \multirow[b]{2}{*}{ LINE } & & \multirow{2}{*}{$\begin{array}{l}\text { No. OF } \\
\text { LINES }\end{array}$} & \multicolumn{2}{|c|}{$W_{\lambda}(\AA)$} & \multirow[b]{2}{*}{ MMF } \\
\hline & & & Arcturus & M13 Stars & \\
\hline $\begin{array}{l}5206.0 \ldots \ldots \ldots \ldots \ldots \\
5269.5 \ldots \ldots \ldots \ldots \ldots \\
5328.0-5328.5 \ldots \ldots \ldots \\
5371.5 \ldots \ldots \ldots \ldots \ldots\end{array}$ & $\begin{array}{l}\mathrm{Cr} \text { I } \\
\mathrm{Fe} \text { I } \\
\mathrm{Fe} \mathrm{I} \\
\mathrm{Fe} \mathrm{I}\end{array}$ & $\begin{array}{l}1 \\
1 \\
2 \\
1\end{array}$ & $\begin{array}{l}0.37 \\
0.70 \\
0.73 \\
0.40\end{array}$ & $\begin{array}{l}0.32 \\
0.45 \\
0.57 \\
0.30\end{array}$ & $\begin{array}{l}0.06 \\
0.17 \\
0.10 \\
0.11\end{array}$ \\
\hline
\end{tabular}

and one which will give insight into the underlying changes, is to consider the behavior of weak $\mathrm{Fe}$ or $\mathrm{Cr}$ lines in Arcturus and in M13 giants of similar $T_{\text {eff }}$. Crude measurements of four such lines were made for several stars in M13 from $4 \mathrm{~m}$ echelle spectra taken by us, while the Arcturus $W_{\lambda}$ are from crude measurements of the Arcturus atlas (Griffin 1968). The results are listed in Table 7, together with the implied MMF values if Arcturus has a $1.1 \mathrm{dex}$ higher metallicity than the M13 stars. The metallicity changes of all these lines except 5269.5 are very small. This is presumably because the flat part of the curve of growth in giants is very long (e.g., Figs. 8-12 of Aller 1963), so that a large abundance change will produce only a slight effect on $W_{\lambda}$. The behavior of the two stronger lines (5269.5 and 5328) is at first sight contradictory, but when one notes that 5328 is actually a blend of two lines, each of which is undoubtedly on the flat part of the curve of growth, its low MMF, when compared with that of 5269.5, is explained. In integrated spectra of galaxies, we therefore expect all features which are blends of individual lines with strengths in the range $0.1-0.4 \AA$ to be fairly insensitive to small metallicity variations. Thus, over the range of metallicity one might expect in galaxies, accuracies presently achievable $\left( \pm 15 \%\right.$ in $\left.W_{\lambda}\right)$ may well prove inadequate to detect any variation in $W_{\lambda}$, even though the abundance of each of the elements with lines in the blend is systematically varying.

The case of the Fe I feature $5270 \AA$ is unique, or almost so, as it is the only weak blend observed here with a single dominant line which is strong enough to be on the damping part of the curve of growth in $\mathrm{K}$ giants. Therefore a more careful treatment of the blend was attempted, using curves of growth appropriate for giants and for dwarfs of varying metallicities. Two predictions of the strength of the $5270 \AA$ Fe I feature were made. In the first (case A) the observations of Pritchet and van den Bergh (1977) were used to interpolate the behavior of the feature for $T_{\text {eff }}<$ $4000 \mathrm{~K}$ (where the last reliable giant and dwarf model atmospheres were available). Thus, below $4000 \mathrm{~K}$, the feature was assumed to increase in strength in dwarfs and decrease in strength in giants. In the second (case B) the feature was assumed to increase to a saturation value of $6 \AA$ in dwarfs and in giants. Above $T_{\text {eff }}=4000 \mathrm{~K}$ for giants and for all dwarfs, the two cases used identical parametrizations for $W_{\lambda}$. The MMF values for the three components used to simulate the $5270 \AA$ blend were also parametrized as functions of $T_{\text {eff }}$ for giants and for dwarfs, based on the $W_{\lambda}$ for $Z=1$ at each $T_{\text {eff }}$ and on the behavior of computed curves of growth at that $T_{\text {eff }}$.

The metallicity sensitivity MS shall be defined as

$$
\mathrm{MS}=\frac{W_{\lambda}(Z=2.0)}{W_{\lambda}(Z=0.5)}-1,
$$

where $W_{\lambda}(Z=2.0)$ is the predicted $W_{\lambda}$ for the feature in the integrated light of the $Z=2.0$ population model, and $W_{\lambda}(Z=0.5)$ is the same for the metalpoor model galaxy. By going from case B to case A in predicting the $5270 \AA$ blend in M giants, MS can be reduced by over $40 \%$. Mould's (1978) calculation followed that of case $B$, except that he did not assume any enhancement of the saturation value in metalrich $\mathbf{M}$ giants, whereas our calculation did. Thus a value of MS determined from his results is intermediate between our case A and case B. Although case B is not consistent with Pritchet and van den Bergh's (1977) observations, it provides the most optimistic possible situation for detecting $\mathrm{Fe}$ metallicity enhancements through the use of the $5270 \AA$ Fe I blend, and in this case for $s=2.35, \mathrm{MS}=0.66$. However, one should note that assumption of a larger drop in the strength of $5270 \AA$ in late $M$ giants than that of case $\mathrm{A}$, which assumed a drop in $W_{\lambda}$ for this feature of $40 \%$ from $T_{\text {eff }}=4000 \mathrm{~K}$ to $T_{\text {eff }}=$ $3000 \mathrm{~K}$ for giants (to give a final MS of 0.37 for $s=2.35$ ), can be made without contradicting the available observational data to produce an even lower value of MS. Thus an observational error of $\pm 18 \%$ in the strength of the $5270 \AA$ blend would suffice to obscure any increase in the strength of the feature as the overall metallicity of the galaxy changed from $Z=0.5$ to $Z=2.0$. The observational material is too fragmentary at this time to say whether the true situation in $\mathbf{M}$ giants is an even steeper dropoff than is assumed for case $\mathrm{A}$. This reduction in MS in case A results from two contributing effects: in the highermetallicity models there is a general shift of the giant branch toward cooler $T_{\text {eff }}$, thus smaller $W_{\lambda}$ in case $\mathrm{A}$, and if one reduces $W_{\lambda}$ in the $\mathrm{M}$ giants, one also reduces the MMF value appropriate for that $T_{\text {eff }}$, as $M M F$, in general, tends to decrease as the line weakens toward the flat part of the curve of growth.

Therefore we emphasize that, at the present time, the observational fact (Faber 1977) that the $5270 \AA$ blend is constant (with an error of $\pm 15 \%$ ) cannot be used to infer that the $\mathrm{Fe}$ abundance is not varying 
systematically between galaxies. Until more observations (now in progress) define the behavior of the $5270 \AA$ feature in $M$ giants, constraints on Fe abundances from the feature at $5270 \AA$ will be impossible to evaluate. Furthermore, if the behavior of the blend is that of case A or worse, as Pritchet and van den Bergh's (1977) observations suggest, observations of very high accuracy $\left( \pm 10 \%\right.$ in $\left.W_{\lambda}\right)$ may well be required to detect variations in the $5270 \AA$ feature due to $\mathrm{Fe}$ abundance variations over the range of metallicities found in galactic nuclei.

\section{d) Problems in the Assumptions}

An area of concern is the effect, as $Z$ is enhanced, of possible cooling of the outer part of the stellar atmosphere where the strong lines are formed and thus of increasing the predicted line strength. This would distort the simple relationships given by equation (1), which was derived without considering this circumstance. Such an effect forms the basis for the proposed explanation offered by Peterson (1976) that super-metal-rich (SMR) stars have merely high CNO abundances. However, this interpretation that SMR stars have only enhanced $\mathrm{C}$ and $\mathrm{N}$ has been challenged by Deming (1978) as well as by the photometry of Gustafsson, Kjaergaard, and Andersen (1974), who believe that SMR stars are really overall metalrich. We shall assume that the latter interpretation of SMR stars is, in fact, correct (see Deming 1978 for a more complete discussion of this controversial point). Furthermore, Hearnshaw (1972) has presented evidence that strong-lined field dwarfs are actually metal-rich. We note that the contribution to the integrated light at $V$ from stars which are not susceptible to possible boundary cooling problems (i.e., from stars near the turnoff and from those giants too cool, according to Peterson, to show the boundary cooling) is very substantial ( $45 \%$ for $\mathrm{Na} \mathrm{D}$ and $80 \%$ for $\mathrm{Mg} b)^{3}{ }^{3}$ Therefore, given that strong-lined dwarfs are actually metal-rich, we do not consider this problem to be serious. One should, however, realize that we have assumed SMR giants are also metal-rich, and furthermore, that differential metallicity-driven boundary cooling, if it occurs, does not substantially affect the strength of the absorption features under consideration.

\section{ABUNDANCES IN GALACTIC NUCLEI}

We now turn to the implications of the observational results of Tables $2 \mathrm{~A}-2 \mathrm{C}$ of line strengths in galactic nuclei. We consider here, if there is a noticeable radial gradient, only the observations at the nuclear pixel, and, in other cases, take the mean of three pixels centered on the nucleus. In Figure 2 we display $W_{\lambda}$ in the nuclei of five galaxies for the strong features plus the three best-observed weaker ones, namely, those in the region of the D lines for which we have the maximum number of frames to average. Estimates of the errors of $W_{\lambda}$ are indicated for $\mathrm{NaD}$ in M32, which are typical of the data, except for the larger errors (also indicated) associated with $\mathrm{Na}$ IR.

A problem would arise if the velocity dispersion variation between the sample galaxies seriously affected the line profile at our resolution and hence the measured $W_{\lambda}$ values. However, the FWHM of $\mathrm{Na} \mathrm{D}$ at the nucleus is $3.4,3.2,4.2,3.6$, and 2.9 pixels,

${ }^{3}$ A different $T_{\text {eff }}$ cutoff for boundary cooling to become unimportant is given by Peterson (1976) for these two lines; thus the contribution is so different in these two cases.

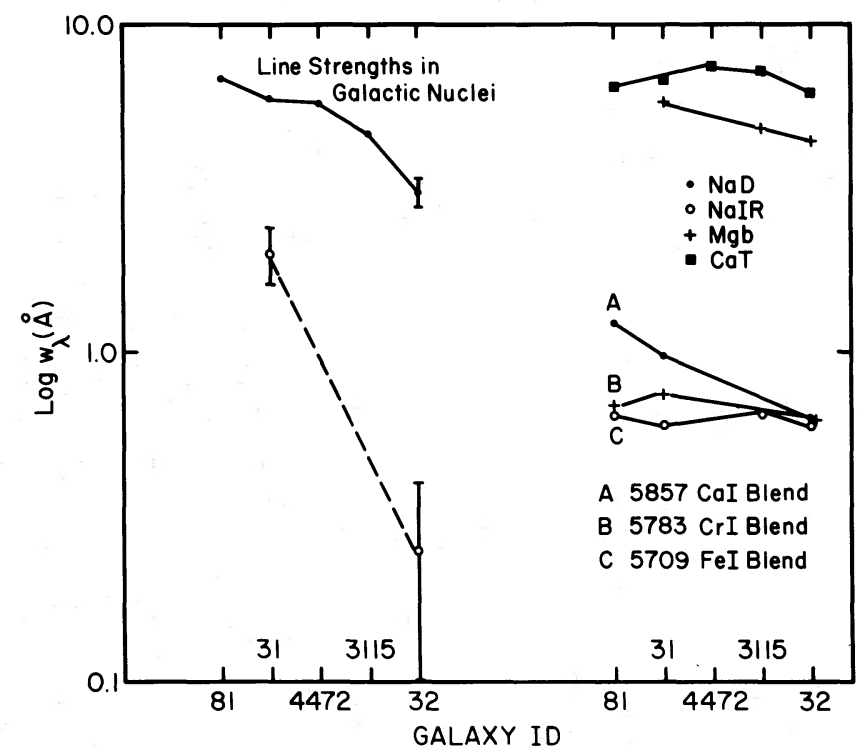

FIG. 2.-The values of the logarithm of $W_{\lambda}$ (in $\AA$ ) for various features are indicated for the nuclei of galaxies. The uncertainties are, in all cases, approximately that shown on the plot for the Na D feature in M32, except that of Na IR where the uncertainty is much larger. 
respectively, for M81, M31, NGC 4472, NGC 3315, and M32. The difference in the nuclear velocity dispersion between M31 and M32 is $80 \mathrm{~km} \mathrm{~s}^{-1}$ (Sargent et al. 1977), while at $5.1 \AA$ pixel $^{-1}$, the corresponding difference is $80 \pm 25 \mathrm{~km} \mathrm{~s}^{-1}$. Thus we may calculate that the total range in velocity dispersion is less than $350 \mathrm{~km} \mathrm{~s}^{-1}$, with all but one of the galaxies within a range of $180 \mathrm{~km} \mathrm{~s}^{-1}$. Furthermore, although the galaxy with the largest velocity dispersion (NGC 4472) does not have strong $\mathrm{Na} \mathrm{D}$, it does have the largest observed $\mathrm{Ca} \mathrm{T}$. Therefore it does not seem that at this resolution the effect of this variation in velocity dispersion will be significant for strong features, although the effect on weaker features may be larger. A more careful modeling of the effects of this parameter is left for future investigations.

We now consider the strengths of the features and compare them to the model predictions of Table 6 . First, we note that $\mathrm{Ca} \mathrm{T}$ is essentially constant at $6.9 \AA( \pm 10 \%)$. Thus the light of these galaxies is, in all cases, giant-dominated with values of $s$ larger than 3.0 eliminated. Because the $\mathrm{Ca} \mathrm{T}$ strength is essentially abundance-independent, it is perhaps more suitable than $\mathrm{CO}$ in determining the value of $s$. Unfortunately, neither has much sensitivity once the integrated light is giant-dominated $(s \lesssim 3)$. Note that the ranking of the galaxies in $\mathrm{Mg} b$ is the same as that of $\mathrm{Na} \mathrm{D}$.

To convert these rankings to a more quantitative abundance, we consider the extreme galaxies M81 (for which observations of only a few spectral features were obtained) and M32 in our line-strength ranking together with M31. Figures $3 a-3 c$ show the ratio of the observed strength of the features in the nuclei (from Tables 2A-2C of this paper and $\mathrm{FeH}$ from Cohen and Phillips 1979) to the predicted strengths as a function of $s$ for $Z=2.0$ and 0.5 . The very large uncertainty in Na IR for M32 is not indicated. By looking for intersections of the lines near $W_{\lambda}^{\text {obs } /}$ $W_{\lambda}^{\text {pred }}=1$, it is clear that the range spanned by these nuclei is from twice to half the solar metallicity, ${ }^{4}$ and that the lines representing all the features intersect at

${ }^{4}$ The metallicity scale is uncertain by the uncertainty in the ACMM calibration, which assumes 47 Tuc to have $Z=0.5$. The range of metallicities for galaxies found here (i.e., a factor of 4) is unaffected by this uncertainty. a single point to within the observational errors. Furthermore, the nonvariation of the 5783 and $5709 \AA$ $\mathrm{Fe}-\mathrm{Cr}$ blends is completely consistent with the above statement (see Table 5 and $\S$ III $c$ ), as is the detectable variation of $\mathrm{Ca}$ I $5857 \AA$ but not of $\mathrm{Ca} \mathrm{T} \mathrm{(a} \mathrm{Ca} \mathrm{II}$ feature). Thus, from the galactic nuclei, we conclude, on the basis of the spectrum from 5000 to $8800 \AA$, that the range in metallicity is from twice (M81, M31) to half solar (M32), and that there is no evidence of any nonsolar ratios among the elements $\mathrm{Na}, \mathrm{Ca}, \mathrm{Mg}$, and $\mathrm{Fe}$ in these $\mathrm{E}$ galactic nuclei. A value of $s$ between 2 and 3 is consistent with all the data.

If we now rank the galaxies by mass or by absolute magnitude (by using the compilation of Burbidge and Burbidge 1975) and compare this to the metallicity ranking of Figure 2, it is apparent that NGC 4472 is anomalous. It is the most massive and the most luminous galaxy of the sample, but it does not have the strongest lines. We will return to this point in $\S \mathrm{V}$, where we will show that this can plausibly be explained by a distance effect, as 1 pixel corresponds to a larger area at this more distant galaxy.

We now consider the published broad-band photometry for these galaxies and apply the metallicity calibration of ACMM to see if the results are consistent with the abundance ranking of Figure 1. Excluding $U$, as spirals and ellipticals are likely to have $U-V$ colors that are not directly comparable due to the presence in the former of large numbers of young stars, the available photometry is summarized in Table 8. The reddening model was taken to be that of Sandage (1973), and the $J-K$ photometry was transformed to the Johnson (1966) system used in ACMM. The expected differences in $V-K$ and $J-K$ colors are not large compared with reddening problems and with problems of correctly matching the $V$ and $K$ apertures, but the data of Frogel et al. (1978) are consistent with the proposed rankings. Furthermore, the nuclear $B-V$ measurements of Sandage, Becklin, and Neugebauer (1969) and Strom et al. (1978), where these two problems are less severe, also support the nuclear metallicity ranking suggested in Figure 1. The nuclear $V-I$ color observed in M31 by Sandage et al. can be reproduced by our $Z=2$ model, which has a predicted $V-I$ of 1.87 for the integrated light,

TABLE 8

UnReddened Galactic Colors*

\begin{tabular}{|c|c|c|c|c|c|}
\hline Galaxy & $\begin{array}{l}(B-V)_{e} \\
(\mathrm{RCBG})\end{array}$ & $B-V_{\text {nuc }}$ & $\begin{array}{c}(V-K)_{0.5} \\
(\text { Frogel et al. } \\
1978)\end{array}$ & $(V-K)_{\text {nuc }}$ & $\begin{array}{c}(J-K)_{0.5} \\
(\text { Frogel et al. } \\
1978)\end{array}$ \\
\hline$\ldots \ldots \ldots \ldots \ldots \ldots \ldots \ldots+$ & 0.94 & $1.10 \dagger$ & $\cdots$ & $3.51 \ddagger$ & . \\
\hline 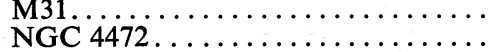 & $\begin{array}{l}0.94 \\
0.95\end{array}$ & $1 .($ & 3.38 & & 0.98 \\
\hline NGC $3115 \ldots$ & 0.98 & $1.00 \S$ & 3.30 & $3.30 \S$ & 1.01 \\
\hline M32. & 0.88 & $0.97 \dagger$ & 3.14 & $\ldots$ & 0.77 \\
\hline
\end{tabular}

* Sandage 1973 reddening-free polar-cap model used in all cases.

$\dagger$ Sandage et al.

$\$$ Aaronson 1977.

§ Strom et al. 1976. 


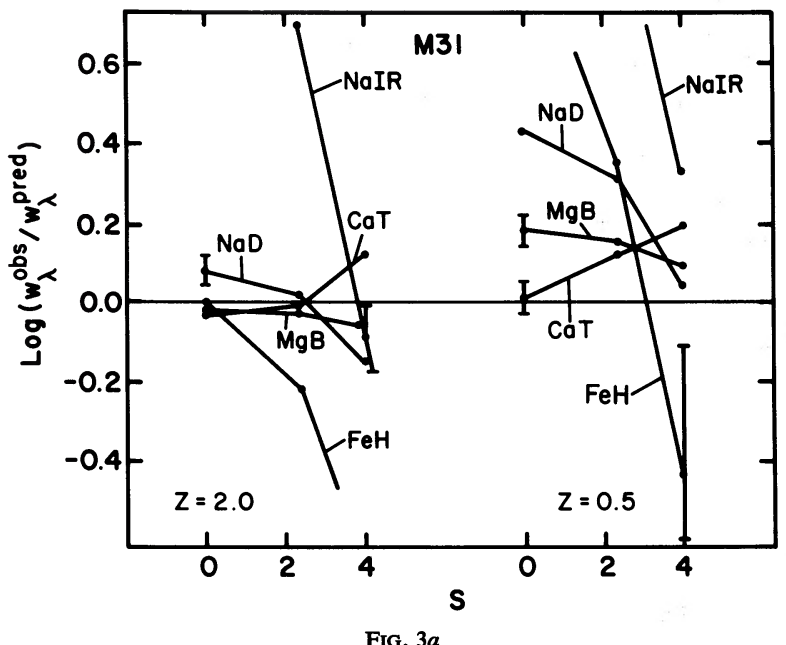

FIG. $3 a$

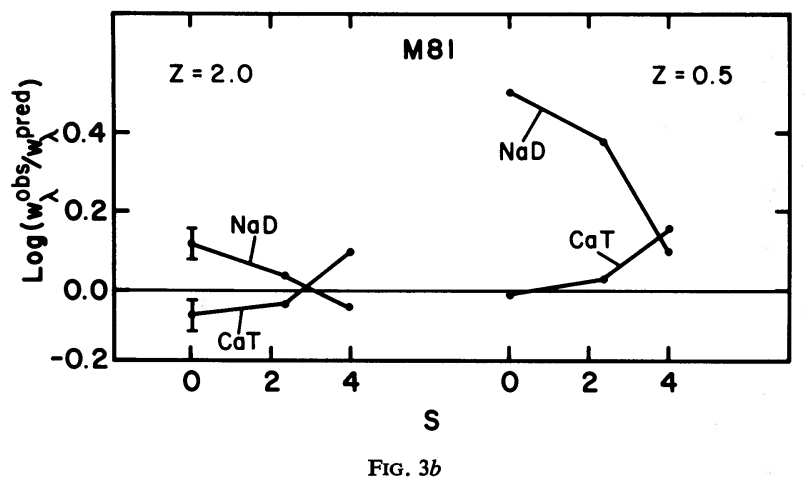

FIG. 3.-(a) The logarithm of ratio of $W_{\lambda}^{\text {obs }}$ to $W_{\lambda}^{\text {pred }}$ is plotted as a function of $s$ (the parameter defining the initial luminosity function) for various features in the spectrum of the nucleus of M31. The $W_{\lambda}^{\text {pred }}$ have been computed for the predicted integrated light for two cases, $Z=2.0$ (a metalrich case) and $Z=0.5$. The observational errors alone are indicated at one point on the line for each feature in either the low or high metallicity case. (b) Same as Fig. $3 a$ but for M81. (c) Same as Fig. $3 a$ but for M32. The very large observational error of Na IR has not been shown.

while the $Z=0.5$ model has a predicted $V-I$ of 1.60 (for $s=2.35$ ). With the use of the ACMM calibration, the change in $V-K$ from M32 to M81 corresponds to an increase from approximately solar to 3 times solar metallicity, also in good qualitative agreement with the change deduced from the spectra via Figures $3 a-3 c$.

\section{RADIAL GRADIENTS IN GALAXIES}

The existence of radial gradients in $W_{\lambda}$ for some, but not all, spectral features is clearly established by the data of Tables $2 \mathrm{~A}-2 \mathrm{C}$. Furthermore, the features which show the strongest radial gradients are those which exhibit the strongest variation between galaxies. We plot in Figure 4 the radial gradients in M31 and $\mathrm{M} 32$ for $\mathrm{Na} \mathrm{D}$ and $\mathrm{Mg} b$. The radial dropoff in $\mathrm{Na} \mathrm{D}$

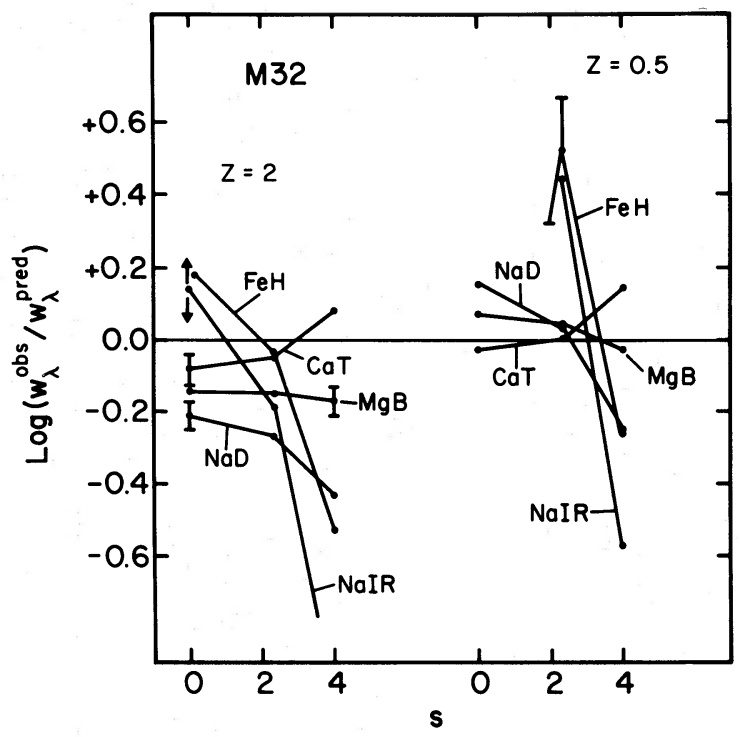

Fig. $3 c$

seen in M31 is in good agreement with the photometric index gradient of Spinrad et al. (1971). As there is only one frame for the $\mathrm{Mg} b$ region, whereas several are available for $\mathrm{Na} \mathrm{D}$, we note that the slope of the radial gradients in M31 and M32 appears to be the same for $\mathrm{Na} \mathrm{D}$, while the slope of $\mathrm{Mg} b$ may be shallower than that of $\mathrm{Na} \mathrm{D}$. It is important to note that in no galaxy is there good evidence for a radial variation of $\mathrm{Ca} \mathrm{T}$ or of the weak $\mathrm{Fe}$ and $\mathrm{Cr}$ blends at 5709 and $5783 \AA$. However, for M31 there is good evidence that $\mathrm{Na}$ IR and the $5857 \AA \mathrm{Ca}$ I blend do have a real radial gradient in $W_{\lambda}$. It is therefore apparent that the fundamental factors driving the radial gradients seen in Figure 4 and Tables $2 \mathrm{~A}-2 \mathrm{C}$ are probably the same as those controlling the changes in nuclear $W_{\lambda}$ seen in Figure 2. Similar conclusions have been reached by Faber (1977). Through our model of $W_{\lambda}$ variations with metallicity developed in $\S$ III, we ascribe all these variations to overall metallicity variations, with no evidence for nonsolar ratios among the elements giving rise to the features studied to date. The less rapid decline in $\mathrm{Mg} b$ than in $\mathrm{Na} \mathrm{D}$ with distance from the nucleus seen in the present data and also those of Faber (1977) is predicted by the theoretical discussion of $\S$ III and is completely consistent with no change in the $\mathrm{Na} / \mathrm{Mg}$ ratio with radius. A variation in metallicity of a factor of 4 over the region from the nucleus to $r=300 \mathrm{pc}$ is derived from Figure 4 and Table 6 based on $\mathrm{Na} \mathrm{D}$. To explore further the hypothesis that the form of the Na Dradius relation is approximately constant in galaxies, as Faber (1977) has suggested, we consider M81 and NGC 3115. In both cases, because of the difference between the distance moduli of the galaxies, 1 pixel in them corresponds to approximately 5 pixels in M31 and M32. The radial gradient in M81 has been plotted in Figure 1 on this scale, and the slope, while slightly shallower, is close to that for M31 and M32. 


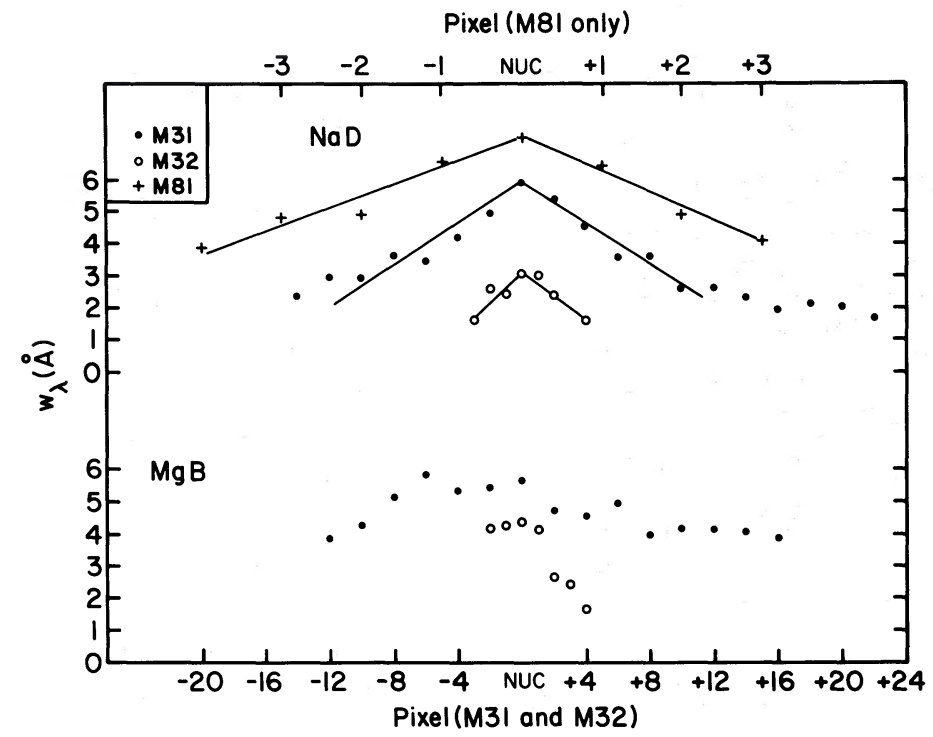

FIG. 4.-Radial variations of $W_{\lambda}$ as a function of pixels from the nucleus for $\mathrm{Na} \mathrm{D}$ and $\mathrm{Mg} b$ are shown for M31 and M32. Radial variations in Na D only are shown for M81 on a scale in pixels (indicated at the top of the plot), corrected for the larger distance of this galaxy. The number of frames available for $\mathrm{Na} \mathrm{D}$ is three for M31 and two for M32, while only one frame is available for $\mathrm{Mg} b$ in these galaxies.

The apparent slight flattening of the curve may well be due to convolution effects, since the spectrum for a point source is 2.5 pixels wide.

The lack of radial variation for $\mathrm{Na} D$ in NGC 4472 and the low nuclear $W_{\lambda}$ are somewhat surprising. Welch and Forrester (1972) saw an enhancement of $\mathrm{Na} \mathrm{D}$ in the central $10^{\prime \prime}$ only, which we did not see because of our poorer spatial resolution. Strom and Strom (1978) find no evidence for a radial color gradient in this galaxy over the region of interest (within $30^{\prime \prime}$ of the nucleus). However, we note that this is the most distant galaxy in our sample. Faber (1977) has suggested that the slope of the radial gradient is constant in $W(\mathrm{Na} \mathrm{D})-\log r$ coordinates, and our data, except for M31, do not extend to large enough radii to test this. In M31 the decrease in $W_{\lambda}$ appears to slow down beyond $r=40^{\prime \prime}$ (see Fig. 4). In this manner the apparent lack of a radial gradient in Na D in NGC 4472 could be understood. We may qualitatively evaluate the effect of such a convolution, assuming a nuclear radial gradient actually exists in $\mathrm{Na} \mathrm{D}$ for this galaxy, within the region covered by the nuclear pixel at a distance 20 times that of M31 to see if the apparently low $W_{\lambda}(\mathrm{Na} \mathrm{D})$ of NGC 4472 can be explained. In Table 9 we indicate the extrapolated nuclear $W_{\lambda}(\mathrm{Na} \mathrm{D})$ at a linear scale of 1 pixel in M31. After this correction, NGC 4472 becomes the galaxy with the most metal-rich nucleus, as was expected from its mass and luminosity (see $\S$ IV). It is apparent that comparison of $W_{\lambda}$ among galaxies (or of photometric indices for spectral features) must be made at the same linear aperture, especially when considering regions near the nucleus. It is unclear how much of the scatter in Figure 2 of Faber (1977) can be due to such effects, as the slope of the radial variations of different features is expected to be different, depending on the composite MMF (i.e., the metallicity sensitivity as defined in $\S$ III $c$ ) for each of the features involved.

Throughout this discussion we have ignored the possible difference between slopes of radial variations along the major and minor axes of the central regions of galaxies, as our data are not adequate for us to comment on this point. Since the major axes of all the galaxies are inclined, with respect to an east-west line, by $52^{\circ} \pm 8^{\circ}$, with the exception of NGC 4472 which is more inclined, a faster minor-axis dropoff will only further increase the nuclear $W_{\lambda}$ for NGC 4472.

We now check the consistency of the radial metallicity variation predicted from our $W_{\lambda}$ measurements with the observed radial color gradients in galaxies. $V-K$ color gradients are given by Strom et al. (1978) along the major and minor axes of NGC 3115. Over the region $r=0^{\prime \prime}-12^{\prime \prime}$, they observe, with a $16^{\prime \prime}$ aperture, a change in $V-K$ of $0.26 \pm 0.05 \mathrm{mag}$ and of $0.46 \pm 0.15 \mathrm{mag}$ from $0^{\prime \prime}$ to $15^{\prime \prime}$. The calibration of ACMM suggests, from the color change, that an overall metallicity change of $0.3-0.5$ dex has occurred

\section{TABLE 9}

EXtrapolated $W_{\lambda}(\mathrm{Na} \mathrm{D})$ FOR Galactic NUCLEI WITH a 15 pc APERTURE

\begin{tabular}{|c|c|}
\hline Galaxy & $(\AA)$ \\
\hline 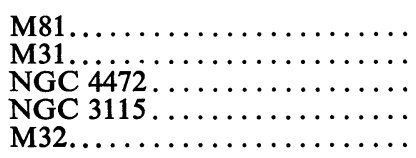 & $\begin{array}{l}7.4 \\
5.9 \\
7.5 \\
5.0 \\
3.1\end{array}$ \\
\hline
\end{tabular}


over this interval, while the $W_{\lambda}$ data for NGC 3115 suggest a change of $0.2-0.3$ dex. We view this agreement as quite reasonable, given the uncertainties involved. It is more difficult to compare the multiaperture photometry of Frogel et al. (1978) with $V-K$ color changes predicted from our observational results, and we note here that only the radial dependence of $V-K$ is in the expected sense, namely, bluer at larger aperture sizes.

\section{CONCLUSIONS}

Observations of the equivalent widths of strong spectral features ( $\mathrm{Ca} \mathrm{T}, \mathrm{Na} \mathrm{D}, \mathrm{Mg} b$, and $\mathrm{Na} \mathrm{IR}$ ) and the weaker $\mathrm{Fe}$ and $\mathrm{Cr}$ blends have been presented for the nuclei of five galaxies (M31, M32, NGC 4472 and 3115, and M81), as well as their radial gradients within these galaxies. These observations have been interpreted with the aid of a model for the strength of spectral features in the integrated light of galaxies which uses the population model of ACMM and is ultimately based on the isochrones of Ciardullo and Demarque (1977). The following is a summary of the important results.

1. The model predicts very small variations of the intermediate-strength Fe blends as the overall metallicity is changed by substantial factors. The observed constancy of all such blends ( $5709 \AA, 5783 \AA$, etc.), with the possible exception of the $5270 \AA$ Fe blend, does not imply nonsolar ratios among the elements heavier than $\mathrm{H}$ and $\mathrm{He}$. The case of the $5270 \AA$ blend is complicated. However, models which fit the available stellar data best indicate that in this case, too, the blend is expected to increase in strength by a factor substantially smaller than previously believed as the overall metallicity of the galaxy changes, so that the variation in $W_{\lambda}$ may be lost in the observational error.

2. The features which vary in strength in these galactic nuclei and which show radial variations are precisely those which the theoretical model has predicted would vary (e.g., $\mathrm{Na} \mathrm{D}, \mathrm{Na} I R, \mathrm{Mg} b$, and the $5857 \AA \mathrm{Ca}$ I blend vary, while $\mathrm{Ca} \mathrm{T}$ and the weak $\mathrm{Fe}$ and $\mathrm{Cr}$ blends do not). The magnitude of the variations is both observed and predicted to be different for various features. Comparison of one feature with another in galaxies of different distance moduli must be done carefully to avoid aperture effects.

3. The features which show radial variations within galaxies are the same as those which show variations among galaxies, while those that do not vary among the nuclei of the galaxies studied also do not show radial variations within these galaxies. The strength of the variations in these two situations is also in agreement. Thus a single causal factor is driving radial variations of spectral features within galaxies and also among galaxies.

4. This causal factor is identified as the overall metallicity of the galaxy. There is no evidence to support nonsolar ratios among the elements $\mathrm{Ca}, \mathrm{Na}$, $\mathrm{Mg}$, and $\mathrm{Fe}$.

5. The form of the variation of $\mathrm{Na} \mathrm{D}$ with radius is approximately the same from galaxy to galaxy in the cases studied.

6. The range in metallicity from M81 to M32 is approximately a factor of 4 . When corrected for the aperture effects, the nuclear metallicity has the same ordering as $M_{v}$ for these five galaxies. Values of $s$ between 2 and 3 are supported by our data.

7. The metallicity gradient within NGC 3115, as predicted from our $W_{\lambda}$ measurements, agrees well with the metallicity gradient predicted by the model of ACMM from the $V-K$ color gradients observed by Strom et al. (1978).

The author is grateful to R. Aikens for his work on the electronic stability of the CID array and to R. Lynds for the use of his software for picture processing.

\section{REFERENCES}

Aaronson, M. 1977, Ph.D. thesis, Harvard University.

Aaronson, M., Cohen, J. G., Mould, J., and Malkan, M. 1978, Ap. J., 223, 824 (ACMM).

Aller, L. H. 1963, Astrophysics: The Atmospheres of the Sun and Stars (2d ed.; New York: Ronald Press), p. 389.

Burbidge, E. M., and Burbidge, G. R. 1975, in Galaxies and the Universe, ed. A. R. Sandage, M. Sandage, and J. Kristian (Chicago: University of Chicago Press), p. 81.

Cayrel, R., and Jugaku, J. 1963, Ann. d'Ap., 26, 495.

Ciardullo, R. B., and Demarque, P. 1977, Trans. Astr. Obs., No. 35 (New Haven: Yale University Press).

Cohen, J. G. 1978, Ap. J., 221, 780.

Cohen, J. G., Frogel, J. A., and Persson, S. E. 1978, Ap. J., $222,165$.

Cohen, J. G., and Phillips, A. C. 1979, in preparation.

Deming, D. 1978, Ap. J., 222, 246.

Deutsch, A. J. 1966, in Abundance Determination in Stellar Spectra, ed. H. Hubenet (London: Academic Press), p. 112.

de Vaucouleurs, G., de Vaucouleurs, A., and Corwin, H., Jr. 1976, Second Reference Catalogue of Bright Galaxies (Austin: University of Texas Press) (RCBG).

de Vaucouleurs, G. 1961, Ap. J. Suppl., 5, 233.
Faber, S. M. 1977, in The Evolution of Galaxies and Stellar Populations, ed. B. M. Tinsley and R. B. Larson (New Haven: Yale University Press), p. 157.

Frogel, J. A., Persson, S. E., Aaronson, M., and Matthews, K. 1978, Ap. J., 220, 75.

Griffin, R. F. 1968, A Photometric Atlas of the Spectrum of Arcturus (Cambridge: Cambridge Philosophical Society).

Gustafsson, B., Kjaergaard, P., and Andersen, S. 1974, Astr. Ap., 34, 99.

Hearnshaw, J. B. 1972, Mem. R.A.S., 77, 55.

Johnson, H. L, 1966, Ann. Rev. Astr. Ap., 4, 192.

Joly, M., and Andrillat, Y. 1973, Astr. Ap., 26, 95.

McClure, R. D. 1969, A.J., 74, 50.

Moore, C. E., Minnaert, M. G. J., and Houtgast, J. 1966, NBS Mono., No. 61 (Washington: NBS).

Mould, J. 1978, Ap. J., 220, 434.

Peterson, R. C. 1976, Ap. J. Suppl., 30, 61.

Pritchet, C., and van den Bergh, S. 1977, Ap. J. Suppl., 34, 101.

Sandage, A. R. 1973, Ap. J., 183, 711.

Sandage, A. R., Becklin, E. E., and Neugebauer, G. 1969, Ap. J., 157, 55. 
Sargent, W. L. W., Schechter, P. L., Boksenberg, A., and Shortridge, K. 1977, Ap. J., 212, 326.

Spinrad, H. S., Gunn, J. E., Taylor, B. J., McClure, R. D., and Young, J. W. 1971, Ap. J., 164, 11.

Spinrad, H. S., Smith, H. E., and Taylor, D. J. 1972, Ap. J., $175,649$.

Strom, K. M., Strom, S. E., Wells, D. C., and Romanishin, W. 1978, Ap.J., 220, 62.
Strom, S. E., and Strom, K. M. 1978, private communication. Strom, S. E., Strom, K. M., Goad, J. W., Vrba, F. J., and Rice, W. 1976, Ap. J., 204, 684.

Tifft, W. A. 1969, A.J., 74, 354.

Tinsley, B. M. 1967, Pub. Dept. Astr., University of Texas at Austin, Ser. 2, Vol. 1, No. 45. - 1972, Ap.J., 178, 319.

Welch, G. A., and Forrester, W. T. 1972, A.J., 77, 333.

Judith G. Cohen: Kitt Peak National Observatory, P.O. Box 26732, Tucson, AZ 85726 